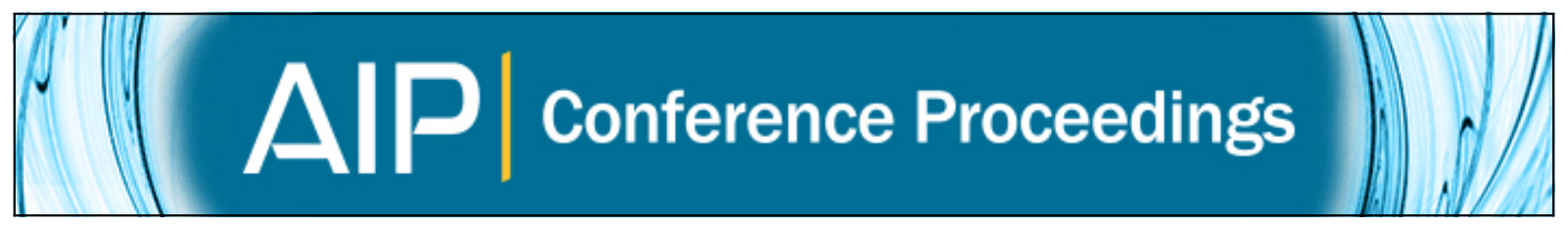

\title{
Effect of doping on the electron transport in polyfluorene
}

Manisha Bajpai, Ritu Srivastava, Ravindra Dhar, and R. S. Tiwari

Citation: AIP Conference Proceedings 1728, 020669 (2016); doi: 10.1063/1.4946720

View online: http://dx.doi.org/10.1063/1.4946720

View Table of Contents: http://scitation.aip.org/content/aip/proceeding/aipcp/1728?ver=pdfcov

Published by the AIP Publishing

Articles you may be interested in

Hot electron effects and nonlinear transport in hole doped manganites

Appl. Phys. Lett. 93, 182110 (2008); 10.1063/1.3020691

Current injection and transport in polyfluorene

Appl. Phys. Lett. 91, 093504 (2007); 10.1063/1.2759951

Dispersive electron transport in an electroluminescent polyfluorene copolymer measured by the current integration time-of-flight method

Appl. Phys. Lett. 79, 2133 (2001); 10.1063/1.1406143

Nondispersive hole transport in an electroluminescent polyfluorene

Appl. Phys. Lett. 73, 1565 (1998); 10.1063/1.122205

Effects of dipolar disorder on electron transport in molecularly doped polymers

J. Chem. Phys. 96, 2390 (1992); 10.1063/1.462036 


\title{
Effect of Doping on the Electron Transport in Polyfluorene
}

\author{
Manisha Bajpai ${ }^{1,3 *}$, Ritu Srivastava ${ }^{2}$, Ravindra Dhar $^{1}$, R .S. Tiwari ${ }^{3}$ \\ ${ }^{1}$ Soft Materials Research Laboratory, Centre of Material Sciences, Institute of Interdisciplinary Studies, University \\ of Allahabad, Allahabad, 211002, India \\ ${ }^{2}$ Physics for Energy Harvesting Division, National Physical Laboratory (Council of Scientific and Industrial \\ Research), Dr K. S. Krishnan Road, New Delhi 110012, India \\ ${ }^{3}$ Department of Physics, Banaras Hindu University, Varanasi-221005, India \\ *Corresponding author email address: mansa83@gmail.com
}

\begin{abstract}
In this paper, electron transport of pure and DMC doped polyfluorne (PF) films have been studied at various doping concentrations. Pure films show space charge limited conduction with field and temperature dependent mobility. The J- V characteristics of doped PF were ohmic at low voltages due to thermally released carriers from dopant states. At higher voltages the current density increases nonlinearly due to field dependent mobility and carrier concentration thereby filling of tail states of HOMO of the host. The conductivity of doped fims were analyzed using the Unified Gaussian Disorder Model (UGDM). The carrier concentration obtained from the fitting show a non-linear dependence on doping concentration which may be due to a combined effect of thermally activated carrier generation and increased carrier mobility.
\end{abstract}

\section{INTRODUCTION}

Polyfluorenes (PFs) are competent materials as blue emitter for white light-emitting PLEDs due to their wide band gap and high photoluminescence efficiency [1]. Hence, for the modeling of PLEDs it is necessary to achieve balanced electron and hole transport in such class of materials. Electron transport in PFO were studied by using time of flight technique and nondispersive electron transport was found in well purified films [2].However, the electron transport becomes highly dispersive by the exposure of films to ambient atmosphere. Electron transports in organic materials are generally found to be affected by trap states. In this work, trap free SCLC has been achieved in PFO for the electron transport by using $\mathrm{Al}$ by doping decamethylcarbonate (DMC) as n-type dopant.

\section{EXPERIMENTAL DETAILS}

Electron only samples of PFO films were prepared in the configuration ITO/PFO: DMC(x wt \%)/Au. ITO sputtered on glass substrates were purchased from Vin Karola, USA. The substrates were patterned and subsequently cleaned ultrasonically and treated with oxygen plasma were used as substrates. Further polymer $(10 \mathrm{mg} / \mathrm{ml})$ dissolved in toluene was deposited onto the precleaned ITO substrates and annealed at $120^{\circ} \mathrm{C}$. Thereafter Gold was thermally vacuum evaporated onto the polymer coated substrates. Electrical measurements were done using Keithley 2610 source measure unit interfaced with computer.

\section{RESULT \& DISCUSSION}

To see the effect of doping on the electrical properties of PFO, single layer device of configuration ITO/PFO: $\mathrm{DMC}(\mathrm{x} \%) / \mathrm{Al}$ were fabricated. We used to choose the same concentrations of DMC for the electrical studies as we 
used for the optical studies. Fig. 1 shows the J-V characteristics of single layer bipolar devices of doped PFO films. It can be seen from the figure that the current density has increased significantly by the doping of DMC in PFO. Current density was found to vary linearly with voltage in the low voltage range $(\mathrm{V}<1 \mathrm{~V})$. This indicates towards an ohmic type conduction mechanism. This mechanism may be attributed to the formation of polaron between PFO and DMC. The conductivity for all the doped devices has been calculated from the ohmic region of Fig 1 and is shown in Fig. 2 as a function of doping concentration. It was recently reported by several authors that the conductivity in doped organic semiconductors is only increased by filling of trap stated followed by free charge carriers [3]. But in our case we have used such system in which LUMO of the polymer $(2.7 \mathrm{eV})$ is just above to the HOMO of the dopant $(3.3 \mathrm{eV})$. This favors a direct electron transfer from dopant to polymer. The charge transfer from dopant to polymer is confirmed from energy level diagram of DMC doped PFO, shown in fig 3. Hence, we have concluded that the increases in

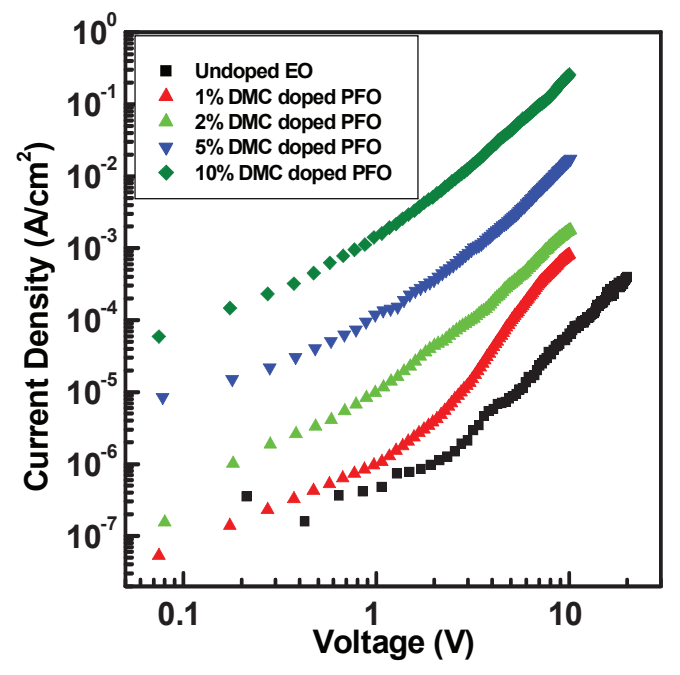

FIGURE1. Current density -voltage characteristics of pristine and DMC doped PFO based electron only devices

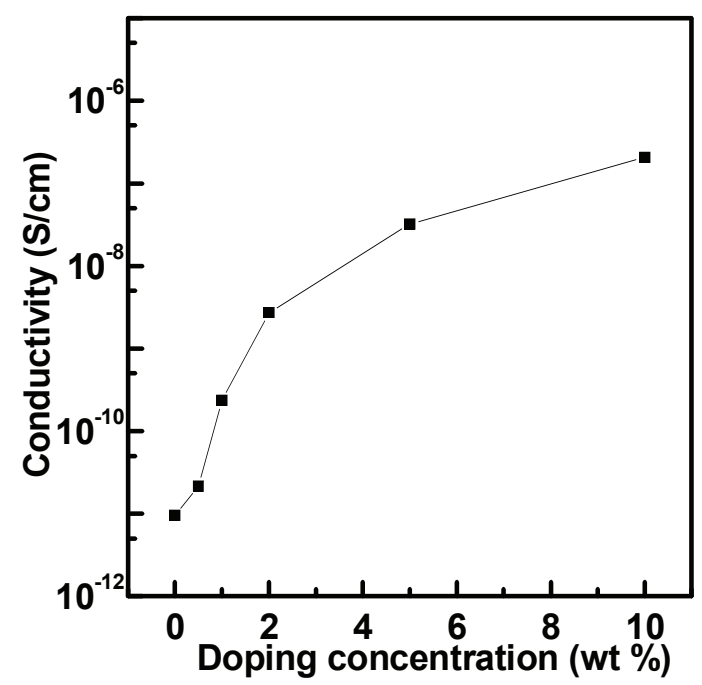

FIGURE 2. Calculated conductivity as a function of doping concentrations 
conductivity in DMC doped PFO system is due to the direct charge transfer generated from the $\mathrm{n}$ type doping of DMC in PFO. Our finding also supports a recent report of Lee et al [4]. They proposed that the doping mechanism of organic semiconductors can be influenced by trap states of host organic semiconductors.

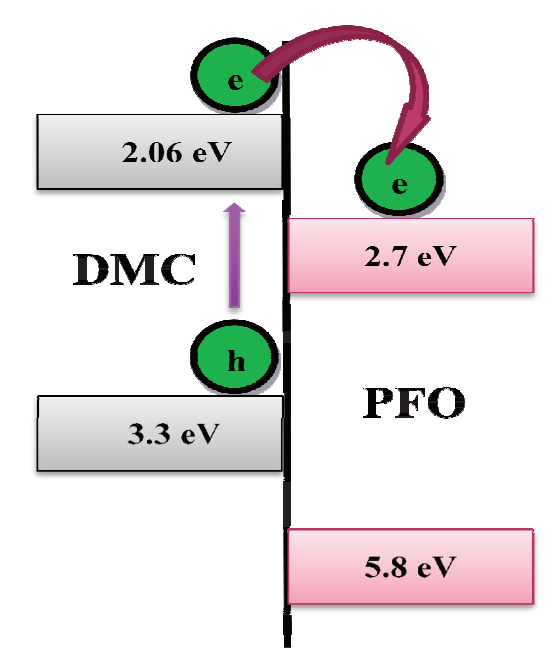

FIGURE 3. Energy level diagram of DMC doped PFO

\section{CONCLUSIONS}

We have created free charge carriers in PFO polymer by using n-type doping of DMC for the amendment of electronic properties. We have electron transport of pristine and doped samples. We have found that the current density increases significantly by the doping of DMC in PFO. Conductivity of PFO was found to be increased from $9.5 \times 10^{-12} \mathrm{~S} / \mathrm{cm}$ to $2 \times 10^{-7} \mathrm{~S} / \mathrm{cm}$ due to the filling of traps.

\section{ACKNOWLEDGEMENT}

The author, Manisha Bajpai gratefully acknowledge the University Grant Commission, New Delhi for the financial assistance under Dr. D S Kothari post doctoral fellowship scheme (F No 4-2 /2006(BSR)/13-998(BSR)).

\section{REFERENCES}

1. M. Bajpai, R. Srivastava, M.N. Kamalasanan, R.S. Tiwari, S. Chand J. Appl. Phys. 109, 084512 (2011).

2. M. Bajpai, R. Srivastava, M.N. Kamalasanan, R.S. Tiwari, S. Chand synthetic Metals, 160, ,1740 (2010).

3. D. F. O. Brien, C. Giebeler, R. B. Fletcher, A. J. Cadby, L. C. Palilis, D. G. Lidzey, P. A. Lane, D. D. C. Bradley, and W. Blau, Synth. Met. 116, 379 (2001).

4. J H Lee, J Lee, Y. H. Kim, C.Yum, B Lussem, K. Leo Org.electron.15,16 (2014). 